\title{
New Measurements for the Resistivity of Curing Period Concrete
}

\section{LU Changguo ${ }^{1, a}$, SUN Bingquan* ${ }^{2, b}$, WANG Jingbo ${ }^{1, c}$, SUN Jiajia ${ }^{2, d}$, XIE $\operatorname{Bin}^{2, e}$}

${ }^{1}$ Department of Mechanical and Electronic Engineering, Yingkou Institute of Technology, Yingkou, Liaoning, China, 115024

${ }^{2}$ Department of Basics, Yingkou Institute of Technology, Yingkou, Liaoning, China, 115024

alucg301@126.com, bhainsbq@126.com, 'jbmail@126.com, ' $15541033881 @ 163 . c o m, ~{ }^{e}$ hellen82@163.com

Keywords: concrete; resistivity; measurements; residual potential.

Abstract. Resistivity measuring can be used as a nondestructive testing technique for concrete. Against the influences of residual potential caused by piezoelectric effect and polarization effect on resistivity measurements, presented a fast, accurate measurement for concrete specimen resistivity, which is, fix the specimens and the electrode plates with a jig, making the contact pressure in between weak, then conduct fast, electrode-switching measurements using digital multimeter, taking the average value as result to eliminate deviation introduced by residual potential. It avoids the trouble of more equipments and wiring of voltammetry, achieving a convenient, simple, fast and stable concrete resistivity testing process. A concrete cube specimen is made according to compressive strength test specification, on which compressive strength tests are conducted directly after resistivity measurements, facilitating the study of relations and rules between resistivity and compressive strength.

\section{Introduction}

The fact that effects the resistivity of concrete include the type of cement, curing conditions, watercement ratio, degree of hydration, relative humidity, aggregate volume fraction and so on [1]. The resistivity of mud is generally much smaller than the aggregate, so concrete can be regarded as a composite material, which is made from an insulator in a conductor based on mud conductive material [2]. In the slurry conductor, the conductive medium is the conductive liquid, and the unhydrated cement and the hydration product is the non-conductive medium. So a hole structure mapping in liquid phase is the formation structure [3]. It has found that the strength of a concrete is related with its resistivity $[1,2,4]$. Concrete resistivity can reflect the full water content in the concrete pores, pore structure of concrete, porosity, pore fluid ion concentration, so it is a very important parameter.

When the electrochemical corrosion reaction happened in the concrete reinforce, the current flowing from the anode to the cathode through in concrete is closely related with its resistivity and the steel corrosion rate. When the ion of a hardened concrete cement slurry flow in its pores, the conductive property is the process of its electrolysis, and the concrete resistivity reflects the potential of concrete reinforcement corrosion rate [5,6]. In recent years, the resistivity of high performance concrete is concerned along with research and application of high performance concrete. The higher concrete performance is, the bigger its resistivity is, because the concrete reinforced protection may affect the result of concrete reinforce. A high-performance concrete wet specimens in the six-month maturity, its resistivity is in the range between $470-530 \Omega \cdot \mathrm{m}$, it's more 10 times than the ordinary concrete, and when the resistivity is more than $500 \Omega \cdot \mathrm{m}$, the steel corrosion in concrete can be greatly suppressed then.

\section{The current ways to measure concrete resistivity and their shortages}

There are many methods for measuring the concrete resistivity, the contact type and the non-contact type are the two main kinds based on its measuring methods [7]. The two electrode method and the four electrode method in the contact type are mostly used at present $[8,9]$. And the non-contact type 
is a new measurement method in recent years. There are more researchers to use. And AC impedance spectroscopy [6] has been applied to the resistance measurement of the cement based materials.

Mr. Gong Guojun and others [10] convert the concrete specimen resistance of $100 \mathrm{~mm} \times 100 \mathrm{~mm}$ $\times 100 \mathrm{~mm}$ cube into specific resistivity data shown in Figure 1 . After the 28 days for standard curing in the ordinary laboratory, then test the specimen's resistance every 20 days. It is a certain applicable method.

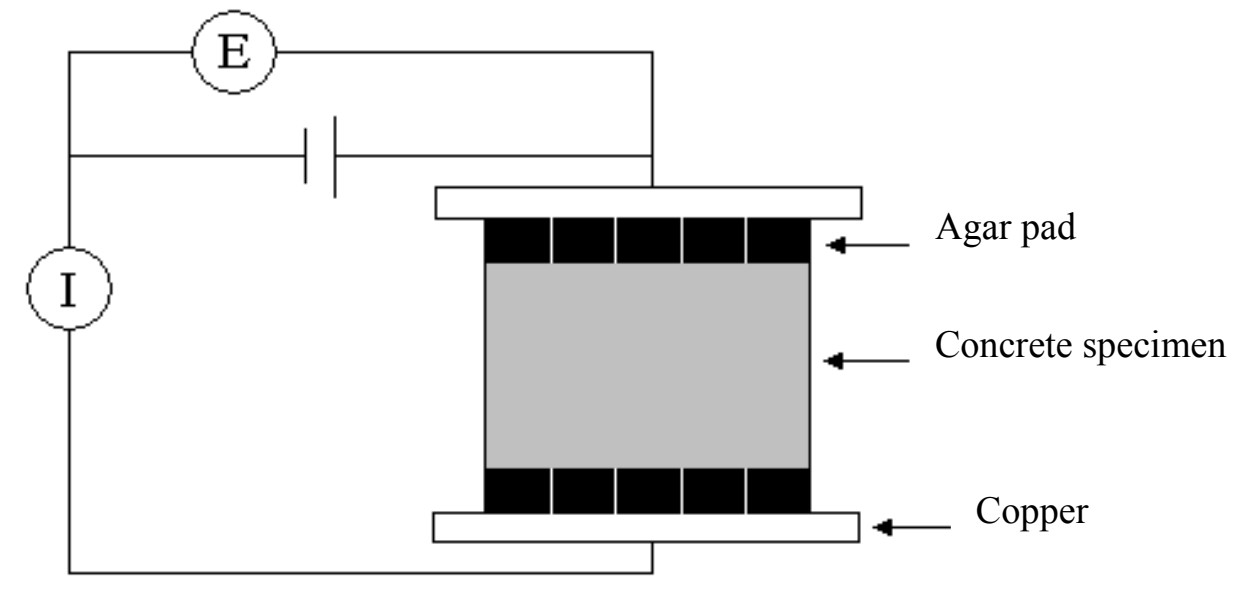

Fig1. The diagram of Measuring Concrete Resistivity

Although so many methods of resistivity measurements and improvements are studied by many researchers and some of them are proposed greatly, but there are still problematic in them [6]: The second electrode method cannot avoid the affect caused by the contact resistance. And the four electrode method can improve the impact of contact resistance, but it is hard to get the electrode ready. And its stability and repeatability are not good, so the results would not be very believable. With AC method to measure is more complex, and the resistivity is variable based on different AC frequency. Non-contact is only to study the early, small sample volume of cement-based materials in the laboratory now, it's not suitable for the actual project online analysis, and must with the help of more sophisticated instruments. And moreover, the equivalent circuit parameters are more complex in AC impedance spectroscopy, must be analyzed based on the mathematical analysis of resistivity data.

\section{A new method for measuring concrete resistivity}

Comparing with other measuring methods above, based on the measuring principle, the one using the two terminal electrodes on the production ends of a concrete block to measure resistance is accurate and feasible; and it is reasonable and necessary for existing concrete to be measured using a four-probe electrode method. Because concrete specimens are uneven ion conductive material, there is a slight residual electric potential, so concrete is also in piezoelectric properties, there will be a certain voltage potential under pressure. It will be a few or some tens of millivolts after tested. It is too small to test with a box bridge measurement method. If tested by voltammetry, the data gained is not good in accurate and repeatable.

In this paper, the bipolar plate is snapped on the opposite surface of the $100 \mathrm{~mm} \times 100 \mathrm{~mm} \times$ $100 \mathrm{~mm}$ (or other dimensions) concrete cube specimens (fixed with a simple jig). Under a constant slight contact pressure, test resistance between the bipolar plate specimen with a digital multimeter. Repeat after reversing two electrodes and take average of the two data. When measuring first test the electrode resistance value R1 with two measuring electrodes contacted with meter ends, then reverse the two specimen electrodes and measure the resistance value $R 2$, if take $R=(R 1+R 2) / 2$ as the test specimen resistance value, it would be within a few seconds. Then the tiny piezoelectric potential and polarization effects are eliminated, and the accuracy and repeatability are in good. This method can avoid the troubles with voltammetry such as more equipment and wiring in use, 
the contact pressure is in convenient and easy. To make specification cubic specimens according to the strength test specification, or a few pieces were tested in long age resistivity nondestructive method, or more specimens in resistance and the pressure intensity value are tested. When doing in such way, we can try to find the resistivity development law in the conservation period. Study and research how it would develop while compressive strength rule varies. The method is as follows:

(1) Curing of concrete specimens resistivity

According to compressive strength test specifications, placed some $100 \mathrm{~mm} \times 100 \mathrm{~mm} \times 100 \mathrm{~mm}$ concrete cube specimens in a drying chamber dried for 1 hour to remove surface moisture after in the conservation of $3 \mathrm{~d}, 7 \mathrm{~d}, 14 \mathrm{~d}$, and the $28 \mathrm{~d}$ respectively, take them out and then measuring their resistance, take the average of three specimens as the data. After that, test their compressive strength directly. We may find the resistivity development law in the conservation period, and the resistivity law related with the compressive strength.

(2) Resistivity measurement method after being long-term placed

When measuring, place the $100 \mathrm{~mm} \times 100 \mathrm{~mm} \times 100 \mathrm{~mm}$ concrete specimens static in lab for 28 days. Then take it up and test its resistivity every $30 \mathrm{~d}$ (or $20 \mathrm{~d}$ ). Take the average value measured of 3 test pieces, keep it in laboratory and control the same temperature between the two tests. The measuring must be repeated at least four times $(30 \mathrm{~d}, 60 \mathrm{~d}, 90 \mathrm{~d}$ and $120 \mathrm{~d}$ ) for studying and researching.

\section{A case of measuring a concrete specimen resistivity in curing}

After testing the resistivity of a sea sand concrete specimens (shown in the Table 1) for study and research, I found that the way taken above is approving, simple and seamless to repeat.

Table 1 The reference concrete mixing proportion $(\mathrm{W} / \mathrm{C}=0.45)$

\begin{tabular}{|cc}
\hline Ingredient & per cubic meter $/ \mathrm{kg}$ \\
\hline Cement & 456 \\
Mixing water & 205 \\
Gravel & 1143 \\
Sand & 563 \\
Slump & $5 \mathrm{~cm}-7 \mathrm{~cm}$ \\
\hline
\end{tabular}

$\mathrm{N}-40$ concrete is made from mixing sand with tap water and to be a standard concrete. And D-40 is from sea sand and water, Df-40 desalination sea sand, water and 15\% fly ash, DL-40 is desalination sea sand and alkaline activated water.

The way I measure resistivity in curing in this paper will be followed below, it can be the operation specification to the long-term placed resistivity measurement method. It is important to operate according to the standard in order to test in the same experimental conditions.

(1) Concrete specimen surface treatment

Clean the surfaces of being placed electrode plate with the brush and alcohol in order to remove the oils and impurity dirt, then dab organic couplant evenly on the surfaces and ensure the electrode plates are well-connected with the them.

(2) The process to make and place electrode plate

It is trouble and inaccurate to place the discharge electrode in the specimen before testing. So we have placed plate electrode on the end faces of concrete specimen in this paper. A $120 \mathrm{~mm} \times 100 \mathrm{~mm}$ election thin sheet of copper (or aluminum, iron tablets etc.) and a $100 \mathrm{~mm} \times 100 \mathrm{~mm}$ auxiliary electrode plate are connected level and evenly, and some plexiglasses are prepare to be used as isolation plate then put and fixed the specimen and electrode plate and auxiliary electrode plate and 
isolation plates together regularly, shown as Figure 2.try to less fixture force if well-connected and keep the force same every time.

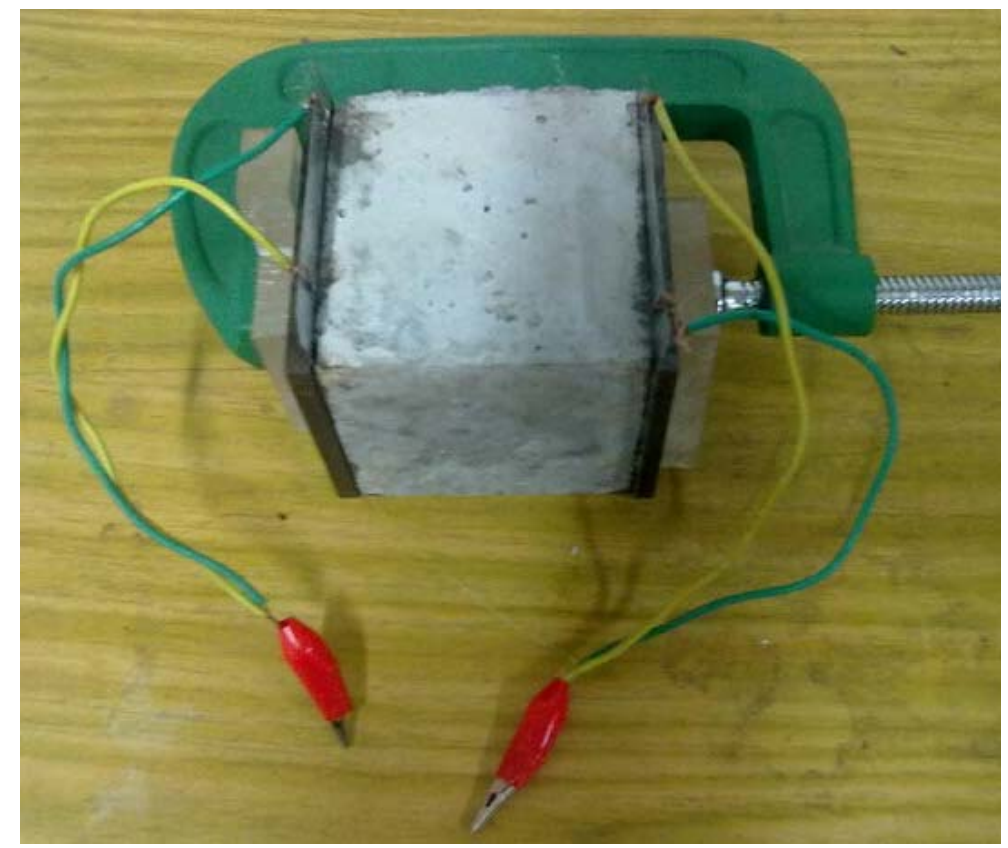

Fig.2 Photo of measuring the electric resistance of concrete

Each concrete sample resistivity is shown in Table 2, the same sample code data is listed in a line in order to compare convenient each other.

Table 2 The compressive Strength and Resistivity of Different Concrete Specimens

\begin{tabular}{lcccccc}
\hline \multirow{3}{*}{ Sample Code } & \multicolumn{3}{c}{ Strength/MPa } & \multicolumn{3}{c}{ Resistivity/k $\Omega \cdot \mathrm{cm}$} \\
& & & & & \\
& & $14 \mathrm{~d}$ & $28 \mathrm{~d}$ & $7 \mathrm{~d}$ & $14 \mathrm{~d}$ & $28 \mathrm{~d}$ \\
\hline C-40 & 31.2 & 35.6 & 41.8 & 11.5 & 12.6 & 13.8 \\
D-40 & 34.0 & 38.6 & 43.5 & 12.8 & 13.5 & 13.6 \\
Df-40 & 32.5 & 37.3 & 41.1 & 10.8 & 11.7 & 12.6 \\
DL-40 & 38.5 & 42.5 & 49.2 & 12.5 & 13.6 & 14.2 \\
\hline
\end{tabular}

It is clear that every specimen's resistivity is bigger than $10 \mathrm{k} \Omega \cdot \mathrm{cm}$ after more than 7 days curing, From Hope [11] finding, the concrete resistivity develop with the curing time. When from $6.5 \mathrm{k} \Omega \cdot \mathrm{cm}$ to $8.5 \mathrm{k} \Omega \cdot \mathrm{cm}$, the inner steel corrosion occurs from possible to impossible. Thereby we come to the conclusion that the inner steel corrosion will not to occur in every specimen. The developing processes of the concrete resistivity and the strength are direct same approximately shown in Table 2. The resistivity of sample DL-40 is high obviously based on its uniformity, density, porosity are better than common tap water in the alkaline activated water concrete.

\section{References}

[1] Hughes B P, Soleit A K O, Brierley R W. New Technique for Determining the Electrical Resistivity of Concrete[J]. Magazine of Concrete Research, 1985, 37(133):243-248.

[2] Wilson J G, Whittington HW, Forde M C. Physical Interpretation of Microcomputer-Controlled Automatic Electrical Resistivity Measurements on Concrete[J]. NDT International, 1985, 18(2):79-84. 
[3] LIU Zhiyong, ZHAN Zhenfeng. Research on electrical resistivity of concrete and its application in durability appreciation of reinforced concrete[J]. Concretes, 2006(10):13-16.

[4] Rengaswamy N S, Srinivaasan S. Non-destructive Testing of Concrete by Electrical Resistivity Measurements [J]. Indian Concrete Journal, 1986, 60(1):23-27.

[5] WEI Xiaosheng, XIA Yuying, WANG Yanwei. Assessment for Chloride Permeability of Concrete by Electrical Resistivity Measurement. Journal of Huazhong University of Science and Technology (Urban Science Edition), 2008, 25(2).

[6] QIAN Jueshi, XU Shanshan, LI Meili, WANG Lixia. The Measurement and Application of Resistivity for Concrete. Journal of Shandong University of Science and Technology (Natural Science), 2010, 29(1).

[7] LI Zhongjin, LI Wenlai. Contactless, transformer-based measurement of the resistivity of materials. US Patent, 6,639,401 B2, 2003.

[8] REZA F, BATSON G B, YAMAMURO J A, et al. Volume electrical resistivity of carbon fiber cement composites[J]. ACI Materials Journal, 2001, 98(1):25-35.

[9] Sihai WEN, and D.D.L. CHUNG. Seebeck effect in carbon fiber-reinforced cement. Cement and Concrete Research 29.12 (1999): 1989-1993.

[10] GONG Guojun, SONG Xiaobing, KONG Qiming. The Resistivity of Concrete Contaminated by Chloride. Industrial Construction, 2005, 35(12).

[11] Hope B B, Ip A K, Manning D G. Corrosion and electrical impedance in concrete[J]. Cement and Concrete Research. 1985, 15(3):525-534. 